\title{
Long-Term Outcomes of Surgical Treatment for Acute Type-A Aortic Dissection with Coronary Artery Involvement
}

\author{
Shuyang Lu, ${ }^{1, *}$ MD, Yun Zhao, ${ }^{2, *}$ MD, Kai Song,${ }^{1, *}$ MD, Wangchao Yao, ${ }^{1}$ MD, Le Kang, ${ }^{1}$ MD, \\ Jun $\mathrm{Li},{ }^{1} \mathrm{MD}$, Yongxin Sun, ${ }^{1} \mathrm{MD}$, Hao Lai, ${ }^{1} \mathrm{MD}$ and Chunsheng Wang, ${ }^{1} \mathrm{MD}$
}

\begin{abstract}
Summary
The surgical strategies for acute type-A aortic dissection (aTAAD) with coronary artery involvement have been controversial, and its prognosis remains unclear. Thus, in this study, we aim to determine the characteristics, surgical strategies, and prognosis of patients with coronary artery involvement due to aTAAD.

Retrospective analysis of 65 consecutive aTAAD patients with coronary artery involvement between September 2005 and January 2012 was performed. The patients were divided into two groups: those treated with aTAAD repair and coronary ostia reimplantation (Neri type-A, group A, $n=37$ ) and those with aTAAD repair and coronary artery bypass grafting (Neri type $\mathrm{B}$ and $\mathrm{C}$, group $\mathrm{B}, n=28$ ).

Overall in-hospital mortality was determined to be $8.1 \%$ for group A and $21.4 \%$ for group B $(P=0.124)$. No significant difference was determined between groups A and B in cardiopulmonary bypass time, cross-clamp time, cerebral perfusion time, and hospitalization time. Intensive care unit (ICU) stay was $5.8 \pm 7.4$ days for group A, whereas it was $12.4 \pm 10.6$ days for group B $(P=0.009)$. The morbidity of postoperative temporary and permanent neurological dysfunction was similar between the two groups, while renal and respiratory dysfunction were $8.1 \%$ versus $25.0 \%$ and $16.2 \%$ versus $39.3 \%$, respectively $(P=0.062, P=0.036)$. Average follow-up time was $112.0 \pm 44.8$ months, and survival curves have not shown statistical significance between two groups $(P=0.386)$.

Coronary artery dissection with Neri type B and C in acute TAAD has been associated with higher early death, but comparable long-term survival after discharge. However, combined immediate coronary artery bypass grafting and aortic repair remains a safe, effective, and acceptable approach to these challenging group of patients.
\end{abstract}

Key words: Neri type, Coronary malperfusion, Coronary artery bypass grafting

(Int Heart J 2021; 62: 1069-1075)

A cute type-A aortic dissection (aTAAD) has been identified to be a catastrophic medical emergency for patients with a high mortality rate if surgery is not performed. According to the International Registry of Acute Aortic Dissection (IRAD), the in-hospital mortality rate for aTAAD patients receiving surgery is as high as $26.6 \%$ versus $55.9 \%$ for those treated with medical therapy alone (overall 32.5\%). ${ }^{1)}$ However, even when aTAAD is identified early and surgery is timely, operative mortality remains high. Early reports of perioperative mortalities after repair of type-A aortic dissection were $30 \%$ to $60 \%$; however, contemporary results have improved because of advances in surgical techniques and improvement of perioperative management, with most recent reports demon- strating a perioperative mortality of $3.09 \%$ to $30 \%{ }^{1-5)}$

Predictors of aTAAD mortality can vary between studies; nonetheless, preoperative status has been demonstrated to be the most important predictor of perioperative hospital mortality. Acute myocardial ischemia or/and infarction due to retrograde dissection of the aortic root reaching the coronary artery ostia is believed to be a potentially fatal condition with poor prognosis. Prompt and optimal interventions to re-establish an adequate coronary blood flow and rescue jeopardized myocardium are recommended for patients with aTAAD with coronary artery involvement. ${ }^{6}$ For peripheral malperfusion, some investigators recommend a strategy of initial percutaneous intervention to restore end-organ perfusion and delayed opera-

From the 'Department of Cardiovascular Surgery, Zhongshan Hospital, Fudan University, The Shanghai Institute of Cardiovascular Diseases, Shanghai, China and ${ }^{2}$ Department of Cardiovascular Surgery, Shanghai Geriatrics Center, Shanghai, China.

*These authors contributed equally to this work.

This study was supported by the National Natural Science Foundation of China (Grant no. 81501595), Youth Foundation of Zhongshan Hospital (Grant no. 2015ZSQN09), Talent Training Program Foundation for the Excellent Youth Supported by Zhongshan Hospital (Grant no. 2017ZSYQ24), Innovation Fund of Zhongshan Hospital (Grant no. 2017ZSCX05, 2020ZSCX07), and Technology Support Project of Science and Technology Commission of Shanghai Municipality of China (20S31901800).

Address for correspondence: Chunsheng Wang, MD, Hao Lai, MD or Yongxin Sun, MD, Department of Cardiovascular Surgery, Zhongshan Hospital, Fudan University, The Shanghai Institute of Cardiovascular Diseases, No. 1609 Xietu Road, Xuhui District, Shanghai 200032, China. E-mail: wang_cs@126.c om or lai.hao@zs-hospital.sh.cn or sun.yongxin@zs-hospital.sh.cn

Received for publication December 27, 2020. Revised and accepted April 12, 2021.

doi: $10.1536 /$ ihj. $20-821$

All rights reserved by the International Heart Journal Association. 
Table I. Patient Demographics and Characteristics

\begin{tabular}{lccc}
\hline Characteristic & Group A & Group B & $P$-value \\
\hline Number of patients & 37 & 28 & \\
Age (years) & $45.4 \pm 9.6$ & $55.1 \pm 10.0$ & 0.000 \\
Gender & & & \\
$\quad$ Male & $29(78.4 \%)$ & $18(64.3 \%)$ & 0.209 \\
$\quad$ Female & $8(21.6 \%)$ & $10(35.7 \%)$ & 0.209 \\
Hypertension & $26(70.3 \%)$ & $22(78.6 \%)$ & 0.451 \\
DM & $7(18.9 \%)$ & $8(28.6 \%)$ & 0.360 \\
Previous CVA & $2(5.4 \%)$ & $3(10.7 \%)$ & 0.426 \\
PVD & 0 & $1(3.6 \%)$ & 0.247 \\
Renal dysfunction & $3(8.1 \%)$ & $6(21.4 \%)$ & 0.124 \\
COPD & 0 & 0 & \\
Previous cardiac surgery & $2(5.4 \%)$ & $1(3.6 \%)$ & 0.727 \\
Previous EVR & 0 & $1(3.6 \%)$ & 0.247 \\
AR & $11(29.7 \%)$ & $7(25.0 \%)$ & 0.673 \\
Marfan syndrome & $1(2.7 \%)$ & 0 & 0.381 \\
BAV & $2(5.4 \%)$ & $2(7.1 \%)$ & 0.773 \\
Acute myocardial infarction & 0 & $2(7.1 \%)$ & 0.099 \\
\hline AR indicates aortic regurgitation; BAV, bicuspid aortic valve; DM, diabetes \\
mellitus; COPD, chronic obstructive pulmonary disease; CVA, cerebrovascular \\
accident; EVR, endovascular repair; and PVD, peripheral vascular disease.
\end{tabular}

tive repair after resolution of malperfusion syndrome. ${ }^{7,8)}$ Girardi, et al. suggest that patients with malperfusion in the setting of aTAAD should undergo immediate aortic reconstruction as the primary means of re-establishing endorgan perfusion. ${ }^{9)}$

As in the treatment of peripheral malperfusion, surgical strategies for aTAAD patients with coronary artery involvement remain controversial, and its prognosis remains unclear. In this present study, we review our experience with aortic dissection involving the coronary arteries and elucidate the characteristics, surgical strategies, and prognosis of this special patient cohort.

\section{Methods}

The protocol of this study was approved by the Committee for the Protection of Human Subjects at Zhongshan Hospital Fudan University (Shanghai, China). Informed consent was obtained from each patient involved in this study. The study design was retrospective and observational.

Patient demographics and characteristics: Between September 2005 and January 2012, we recruited 65 patients with coronary artery involvement out of the 267 patients who had undergone surgical repair of aTAAD. The mean age was $49.6 \pm 10.8$ years; age range was $26-70$ years old, and 47 were male. The acute phase of aortic dissection was defined as being within 2 weeks from symptom onset to the institution of therapy. Preoperative diagnoses of TAAD were based on the computed tomography angiography and echocardiography results. Types of coronary ostial lesions were described by Neri, et $a$., and they are as follows: type A, ostial dissection defined as a disruption of the inner layer limited to the area of the coronary ostium; type $\mathrm{B}$, dissection with a coronary false channel; and type $\mathrm{C}$, circumferential detachment with an inner cylinder intussusception. ${ }^{6}$ In addition to the three patients who had preoperative electrocar- diographic signs of myocardial ischemia, all aTAAD patients with coronary artery involvement were testified by the results of the surgical exploration. Among them, 37 patients with mild coronary artery involvement (Neri typeA) were treated with aTAAD repair and coronary ostia reimplantation (group A), whereas 28 patients with moderate to severe coronary artery involvement (Neri type B and $C$ ) were treated with aTAAD repair along with coronary artery bypass grafting (group B).

Although the differences were not statistically significant, preoperative hypertension, diabetes mellitus (DM), and renal dysfunction were more common in the patients of group B $(78.6 \%$ versus $70.3 \%, P=0.451,28.6 \%$ versus $18.9 \%, P=0.360$, and $21.4 \%$ versus $8.1 \%, P=0.124$, respectively). No significant differences were noted in other preoperative medical disorders between the two groups. Further demographic and preoperative data are summarized in Table I.

Operative technique: Our approach for open aortic arch reconstruction has been described previously in detail. ${ }^{10,11)}$ All patients were carried out by a median sternotomy and total cardiopulmonary bypass (CPB) with selective antegrade cerebral perfusion (ACP). The left radial artery and dorsal artery of foot were cannulated for continuous blood pressure monitoring. Temperature probes were placed for esophagopharyngeal and bladder temperature monitoring. Right axillary artery and right femoral artery were routinely used for arterial perfusion cannulation. Circulatory arrest was initiated when the nasopharyngeal temperature reached $17^{\circ} \mathrm{C}$ to $22^{\circ} \mathrm{C}$ and bladder temperature reached $26^{\circ} \mathrm{C}$ to $28^{\circ} \mathrm{C}$. Pharmacological agents (thiamylal sodium, phenytoin, and mannitol) were then administered for brain protection in addition to ice packs placed around the head. The right axillary artery was used for selective ACP during arch reconstruction. The flow rate was maintained at 8 $\mathrm{mL} / \mathrm{kg} /$ minute to $10 \mathrm{~mL} / \mathrm{kg} /$ minute and the perfusion pressure at $40 \mathrm{mmHg}$ to $50 \mathrm{mmHg}$.

Open aortic arch reconstruction techniques for this 
Table II. Surgical Strategy

\begin{tabular}{lccc}
\hline Variables & Group A & Group B & $P$-value \\
\hline Extent of aortic procedure & & & \\
AAR+HAR & $9(24.3 \%)$ & $5(17.9 \%)$ & 0.530 \\
AAR+TAR (4-branch prosthetic graft) & $14(37.8 \%)$ & $7(25.0 \%)$ & 0.273 \\
AAR+TAR (island anastomosis) & $13(35.1 \%)$ & $15(53.6 \%)$ & 0.137 \\
AAR+Triple-branched stent graft implantation & $1(2.7 \%)$ & $1(3.6 \%)$ & 0.841 \\
Elephant trunk technique & $21(56.8 \%)$ & $20(71.4 \%)$ & 0.225 \\
Concomitant procedures & & & \\
Ascending aorta replacement & $37(100 \%)$ & $28(100 \%)$ & \\
Bentall operation (mechanical valve) & $30(81.1 \%)$ & $8(28.6 \%)$ & 0.000 \\
David operation & $5(13.5 \%)$ & $4(14.3 \%)$ & 0.929 \\
Cabrol operation & $2(5.4 \%)$ & 0 & 0.211 \\
Wheat operation & 0 & $3(10.7 \%)$ & 0.041 \\
Aortic root reinforcement & 0 & $13(46.4 \%)$ & 0.000 \\
CABG (SVG) & & & \\
RCA & 0 & $23(82.1 \%)$ & 0.000 \\
LAD & 0 & $5(17.9 \%)$ & 0.007 \\
\hline AAR indican
\end{tabular}

AAR indicates ascending aorta replacement; CABG, coronary artery bypass grafting; HAR, hemi-arch replacement; RCA, right coronary artery; SVG, saphenous vein graft; and TAR, total arch replacement.

cohort of patients included total arch replacement (TAR) with four-branch, one-branch, or straight prosthetic graft for island anastomosis, along with a stent graft (MicroPort Medical Co Ltd., Shanghai, China); triple-branched stent graft implantation technique (Yuhengjia Sci Tech Corp Ltd., Beijing, China) for TAR; and hemiarch replacement (HAR). The arch reconstruction strategy was mainly based on intraoperative inspection, including location of the primary tear, extent of the tear, vascular-wall texture, and situation of the supra-arch vessels.

The proximal aortic root procedures were performed during cooling and the systematic rewarming duration and based on the surgical inspection of the involvement of the aortic root, including the aortic valve leaflets as well as the coronary ostia. For the patients of group A, coronary artery ostia involvement was deemed not serious, and coronary artery reimplantation was performed to avoid coronary artery bypass grafting. Specific techniques for aortic root reconstruction of the present group included Bentall, David, and Cabrol surgery. For the patients of group $\mathrm{B}$, the severity of coronary ostia involvement reached Neri type $B$ and $C$, and intima was fragile and detached with adventitia. All patients of this group underwent coronary artery bypass grafting. Specific aortic root techniques of this group included Bentall, David, Wheat, and aortic root reinforcement surgery. ${ }^{11)}$

Statistical analysis and follow-up: Data were collected from chart review and were entered into a dedicated Microsoft Excel table. Categorical variables are represented as frequency distributions and single percentages. Values of continuous variables are expressed as mean \pm standard deviation. Survived patients were followed yearly in our outpatient department or by phone calls to evaluate their clinical status. Normally distributed continuous variables were compared using Student t-test, non-normally distributed continuous variables using Mann-Whitney $U$ test, and categorical variables using $\chi^{2}$ and Fisher's exact test, where appropriate. Long-term survival analysis was conducted using Kaplan-Meier method with log-rank test for group comparisons. All statistical tests were two-sided. Results were considered statistically significant at a level of $P$ less than 0.05 . All analyses were performed with the IBM SPSS statistical package version 26.0 (IBM Corp. Armonk, NY, USA).

\section{Results}

Perioperative data: Table II shows the extent of aortic repair and concomitant procedures in both groups. The ascending aorta was replaced in all patients. Most patients of both group A and B underwent TAR. However, more island anastomosis operations were performed in group B (35.1\% versus $53.6 \%, P=0.137$ ), while more four-branch prosthetic grafts were used in group A $(37.8 \%$ versus $25.0 \%, P=0.273)$. Composite graft replacement of the aortic root (Bentall procedures) had been extensively applied in group A as compared to group B $(81.1 \%$ versus $28.6 \%, P<0.001$ ), while $46.4 \%$ of patients of group B underwent aortic root reinforcement operations and avoided Bentall or David operations. In group B, all patients underwent coronary artery bypass grafting (CABG) for Neri type $\mathrm{B}$ and $\mathrm{C}$ lesions. There were 23 patients $(82.1 \%)$ who needed right coronary artery bypass, while only 5 patients $(17.9 \%)$ needed left coronary artery bypass. Other procedures of the David operation, hemiarch replacement, triple-branched stent graft implantation, and elephant trunk technique showed no significant difference between the two groups.

As shown in Table III, there were no significant differences in terms of CPB time $(P=0.990)$, cross-clamp time $(P=0.882)$, cerebral perfusion time $(P=0.325)$, overall in-hospital stay $(P=0.179)$, packed red blood cell transfusions $(P=0.118)$, serum transfusions $(P=0.627)$, and volume of drainage during the first postoperative day $(P=0.216)$. Patients in group $\mathrm{B}$ had significantly longer ICU stay $(P=0.009)$ and ventilation time $(P=0.025)$.

In-hospital mortality and morbidity: As shown in Table IV, overall in-hospital mortality and 30-day operative mor- 
Table III. Perioperative Data

\begin{tabular}{|c|c|c|c|c|c|}
\hline & \multicolumn{2}{|c|}{ Group A } & \multicolumn{2}{|c|}{ Group B } & \multirow[b]{2}{*}{$P$-value } \\
\hline & Mean $\pm \mathrm{SD}$ & $\begin{array}{c}\text { Range } \\
\text { percentage }\end{array}$ & Mean $\pm \mathrm{SD}$ & $\begin{array}{c}\text { Range } \\
\text { percentage }\end{array}$ & \\
\hline CPB time (minutes) & $177.7 \pm 58.4$ & 94-307 & $177.5 \pm 44.5$ & $115-303$ & 0.990 \\
\hline Cross-clamp time (minutes) & $103.9 \pm 33.4$ & $60-184$ & $102.5 \pm 40.7$ & $34-178$ & 0.882 \\
\hline Cerebral perfusion time (minutes) & $34.5 \pm 15.5$ & $10-73$ & $31.0 \pm 11.1$ & $18-55$ & 0.325 \\
\hline Nasopharyngeal temperature $\left({ }^{\circ} \mathrm{C}\right)$ & $17.8 \pm 2.7$ & $11.9-23.3$ & $18.2 \pm 1.7$ & $14.6-20.8$ & 0.525 \\
\hline Rectal temperature $\left({ }^{\circ} \mathrm{C}\right)$ & $21.1 \pm 2.5$ & $17.0-25.3$ & $22.7 \pm 2.4$ & $17.7-28.0$ & 0.013 \\
\hline In-hospital stay (days) & $19.6 \pm 13.3$ & $1-59$ & $23.7 \pm 10.0$ & $2-41$ & 0.179 \\
\hline ICU stay (days) & $5.8 \pm 7.4$ & $1-40$ & $12.4 \pm 10.6$ & $1-39$ & 0.009 \\
\hline Ventilation time (hours) & $75.3 \pm 168.0$ & $8-960$ & $193.6 \pm 214.4$ & $11-936$ & 0.025 \\
\hline Transfused RBC (mL) & $1439.7 \pm 1351.5$ & $400-7500$ & $1948.1 \pm 1047.8$ & $200-5000$ & 0.118 \\
\hline Transfused serum (mL) & $1452.9 \pm 1500.1$ & $400-9000$ & $1607.7 \pm 672.9$ & $400-3000$ & 0.627 \\
\hline Platelet (patients, \%) & 13 & $35.10 \%$ & 11 & $39.30 \%$ & 0.731 \\
\hline Platelet (pack) & $0.5 \pm 0.8$ & $0-4$ & $0.5 \pm 0.7$ & $0-2$ & 0.832 \\
\hline Drainage of first day $(\mathrm{mL})$ & $563.0 \pm 402.9$ & $210-1600$ & $438.5 \pm 252.2$ & $115-1170$ & 0.216 \\
\hline
\end{tabular}

CPB indicates cardiopulmonary bypass; ICU, intensive care unit; and RBC, red blood cell.

Table IV. In-Hospital Mortality and Morbidity

\begin{tabular}{lccc}
\hline & Group A & Group B & $P$-value \\
\hline In-hospital mortality & $3(8.1 \%)$ & $6(21.4 \%)$ & 0.124 \\
Operative mortality within 30 days & $2(5.4 \%)$ & $3(10.7 \%)$ & 0.426 \\
Acute renal dysfunction & $3(8.1 \%)$ & $7(25.0 \%)$ & 0.062 \\
Respiratory dysfunction & $6(16.2 \%)$ & $11(39.3 \%)$ & 0.036 \\
Redo for bleeding & $2(5.4 \%)$ & $1(3.6 \%)$ & 0.727 \\
Mediastinum infection & $1(2.7 \%)$ & $1(3.6 \%)$ & 0.841 \\
TND & $2(5.4 \%)$ & $1(3.6 \%)$ & 0.727 \\
PND & $3(8.1 \%)$ & $2(7.1 \%)$ & 0.885 \\
\hline
\end{tabular}

PND indicates permanent neurological dysfunction; TND, temporary neurological dysfunction.

tality were $8.1 \%, 5.4 \%$ for group A and $21.4 \%, 10.7 \%$ for group $\mathrm{B}$, respectively $(P=0.124,0.426)$. In group $\mathrm{A}$, causes of in-hospital death were as follows: multiple organ failure with permanent neurological dysfunction, respiratory dysfunction, renal dysfunction, and massive gastrointestinal bleeding in one patient, permanent neurological dysfunction, and respiratory dysfunction in two. In group B, causes of in-hospital death were as follows: multiple organ failure in one patient, low cardiac output syndrome in two, and sepsis in one.

The morbidities of postoperative acute renal dysfunction and respiratory dysfunction were observed to be more common in group B, although there was no statistical difference for renal dysfunction. Acute renal dysfunction was observed in 7 of 28 patients in group B and in 3 of 37 patients in group A $(P=0.062)$. Respiratory dysfunction was observed in 11 of 28 patients in group B and in 6 of 37 patients in group A $(P=0.036)$. The morbidities of temporary neurological dysfunction, permanent neurological dysfunction, redo sternotomy for bleeding, and mediastinal infection were similar in the two groups $(P=$ $0.727, P=0.885, P=0.727, P=0.841$, respectively).

Follow-up and long-term outcomes: Average follow-up time was $112.7 \pm 53.6$ months for group $\mathrm{A}$ and $111.1 \pm$ 31.2 months for group B $(P=0.898)$. Survival curves (excluding in-hospital deaths) of both surgical techniques showed no statistically significant difference between group A and group B (log-rank $P$-value $=0.386$, Figure) Median survival time was $131.8 \pm 11.9$ months $(95 \% \mathrm{CI}$, 108.4-155.3) for group A and $144.2 \pm 8.8$ months $(95 \%$ CI, 126.9-161.5) for group B.

As shown in Table V, overall mortality was $20.6 \%$ for group A and $13.6 \%$ for group B during the follow-up $(P=0.507)$. In group A, early causes of death were cerebral hemorrhage due to warfarin overdose in three patients and infection with secondary descending aortic dissection rupture in one patient, while late causes of death were secondary abdominal aortic dissection rupture in one patient and carcinoma of respiratory and digestive system in two patients. In group B, there were one early death due to warfarin overdose and two late deaths due to rectal cancer and secondary rupture of abdominal aortic dissection. There were no significant differences in complications of cerebral hemorrhage, secondary descending aortic dissection rupture, and cancer, respectively.

\section{Discussion}

Acute TAAD has been identified as a life-threatening disease due to its high mortality and morbidity. Once diagnosed, emergent or urgent surgical intervention is required. If aTAAD is concomitant with acute coronary artery malperfusion or myocardial infarction, the condition is more complicated, and the mortality rate is higher. In 


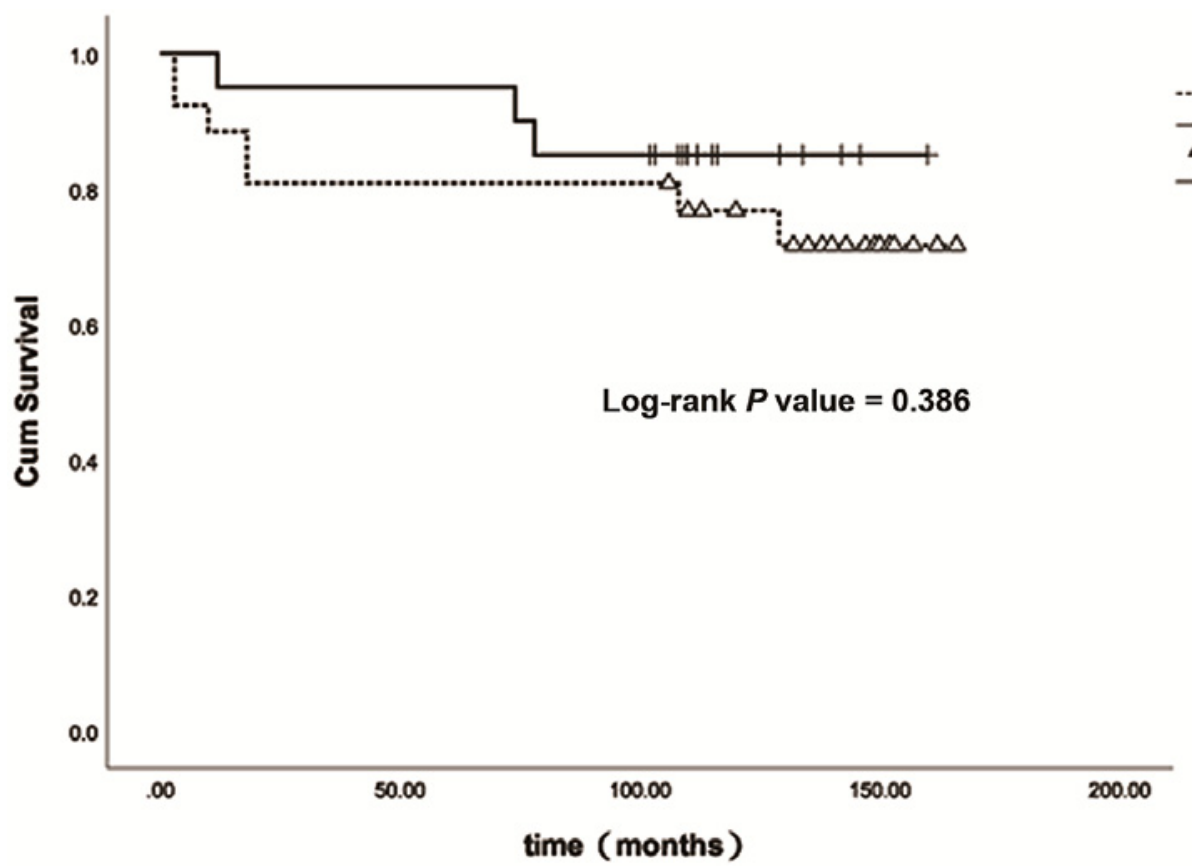

Figure. The survivor functions (excluding in-hospital deaths) were not significantly different between group A and group B (Logrank $P$-value $=0.386$ ).

Table V. Follow-Up and Long-Term Outcomes

\begin{tabular}{lllc}
\hline & Group A & Group B & $P$-value \\
\hline Overall mortality & $7(20.6 \%)$ & $3(13.6 \%)$ & 0.507 \\
Early death (within 24 months) & $4(11.8 \%)$ & $1(4.5 \%)$ & 0.355 \\
Late death & $3(8.8 \%)$ & $2(9.1 \%)$ & 0.973 \\
Cerebral hemorrhage & $3(8.8 \%)$ & $1(4.5 \%)$ & 0.544 \\
Descending aortic dissection rupture & $2(5.9 \%)$ & $1(4.5 \%)$ & 0.828 \\
Cancer & $2(5.9 \%)$ & $1(4.5 \%)$ & 0.828 \\
\hline
\end{tabular}

this study, we systematically summarized the characteristics, surgical strategies, and prognosis of patients with acute TAAD with coronary artery involvement.

For characteristics of aTAAD patients with coronary involvement, Chen, et al. found that patients with acute coronary involvement because of TAAD are much younger (51.8 \pm 11.8 years), have a lower incidence of a history of hypertension, and have a higher aortic regurgitation rate. ${ }^{12)}$ In our study, patients of group B were much older $(55.1 \pm 10.0$ years) with a high rate of hypertension (78.6\%); on the contrary, patients of group A were much younger (45.4 \pm 9.6 years) with a relative low rate of hypertension $(70.3 \%)$. Note that hypertension is the most common risk factor for aTAAD. We have also found that incidence of preoperative renal dysfunction of group B was much higher than group A $(21.4 \%$ versus $8.1 \%)$. It might correlate with the severity of coronary artery involvement and advanced age, which indicate poor prognosis. ${ }^{5)}$

The overall rate of aTAAD with coronary artery involvement is not low, and the proportion of cases in this group is as high as $24.3 \%$ (65/267). In most cases, although the coronary ostia are involved, there is no myocardial ischemia, so it is difficult to make a definite diag- nosis before operation. Unless the patient has abnormal changes in the electrocardiogram, or the degree of coronary artery involvement is serious, cardiac echocardiography can catch sight of coronary involvement. In group A, the coronary artery ostia of all patients were slightly involved, and there were no myocardial ischemia manifestations before surgery. All of the diagnoses were confirmed during surgery. Only three patients in group B had myocardial ischemia before operation. Two of them belonged to Neri $\mathrm{C}$ type with coronary artery intimal rupture. On the whole, preoperative electrocardiogram (ECG) showed that only $4.6 \%$ of all cases were diagnosed with coronary artery involvement $(3 / 65)$. In literatures, the incidence of acute myocardial ischemia due to type-A aortic dissection has been reported at $5.7 \%$ to $11.3 \%$, which is higher than our incidence. ${ }^{6,13)}$ Therefore, although aTAAD involves a high proportion of coronary artery ostia affected, the cases of serious myocardial ischemia are not high. However, its true clinical significance is that it makes aortic root reconstruction become more complex.

The surgical outcomes of TAAD have been dramatically improved in recent years. Sun, et al. reported the treatment of 291 patients with TAAD with total aortic arch replacement with stented elephant trunk implantation, 
with in-hospital mortality only at 3.09\%. ${ }^{3)}$ However, myocardial damage caused by coronary dissection is one of the leading predictors of hospital death. Coronary ischemia and the need for concomitant CABG have been reported to be the independent risk factors for mortality. ${ }^{5,13)}$ On the one hand, insufficient coronary perfusion may increase the difficulty of $\mathrm{CPB}$ weaning and prolong the $\mathrm{CPB}$ time, exacerbating complement-mediated inflammatory response. Additionally, myocardial ischemia triggers inflammatory "waterfall" responses, thus increasing the incidence of acute respiratory distress syndrome, myocardial stunning, and so on, leading to an increase in mortality. ${ }^{14,15)}$ Eren, et al. reported there were three $(21.4 \%)$ early postoperative deaths for aTAAD patients with coronary involvement in their study, and this mortality rate was significantly worse than that in patients without coronary involvement over the same period of study, having only mortality rate of $13.4 \%(17 / 122) .{ }^{13)}$ In our study, there were three patients with preoperative electrocardiogram indicating myocardial ischemia, and two of these patients died of multiple organ failure after active treatment. These results are enough to demonstrate the perioperative risk of myocardial ischemia/infarction for aTAAD.

Neri, et al. reported that they preferred regional repair of torn coronary arteries to $\mathrm{CABG}$ out of technical and hemodynamic considerations. ${ }^{6}$ They thought of the following advantages: (1) reconstruction of the coronary ostia was anatomic, and it avoided extensive revascularization on vessels whose diameter, importance, and status were often undetermined; (2) it avoided ligature of the coronary ostium and the consequent complete graftdependent perfusion of large territories of the myocardium; and (3) it provided antegrade flow in the coronary tree, thus avoiding the risk of competitive flows and of coronary re-dissection. However, repairing the coronary artery is usually cumbersome and time-consuming since the tissues are dissected and very fragile. We instead prefer grafting the distal coronary due to the simplicity of the procedure, which would also facilitate prompt cardioplegia delivery to protect the affected myocardium via the bypass graft. ${ }^{16,17)}$ For the patients of group A with Neri type-A lesion, we agreed with Neri, et al. and performed anatomic reconstruction of coronary ostia. Composite graft replacement of the aortic root for reimplantation of the restored coronary orifices (Bentall procedures) has been extensively applied in this series mostly because of the following reasons: patients are much younger and high incidence of commissural detachment. For the patients of group B with Neri type B and C lesions, we prefer to perform CABG with aortic valve-sparing techniques or aortic root reinforcement techniques when possible. The patients in this series are much older, and preoperative status is complex. The goal of the operation should be to solve the patient's main problems with the simplest and most effective methods.

The follow-up results show that although the inhospital mortality of the patients in the group A was lower than that in the group $\mathrm{B}$, there were no significant differences in terms of the early and late mortalities. There was also no significant difference between the two groups as regards the causes of the patients' follow-up death. However, patients of group A developed more cerebral hemorrhage due to warfarin overdose, which was closely related to the higher proportion of mechanical valve use in previous surgery.

Limitations: This present study has some inevitable limitations. First, this is a retrospective observational study, and the nonrandomized design may have affected the results, through unrecognized confounding factors and bias. Second, this study represents only a single-center experience, and not a multi-center one. There surely are some disparities among different centers and surgeons. Finally, with the advances in surgical techniques, anesthesia, cerebral protection, and perioperative care, they surely affect the surgical results of different periods.

\section{Conclusions}

Coronary artery dissection with Neri type B and C in acute TAAD has been found to be associated with higher early death, but comparable long-term survival after discharge. However, there are several factors that can affect the outcome, such as advanced age, more renal dysfunction, and worse preoperative conditions. Immediate CABG and aortic repair remains to be a safe, effective, and acceptable approach to these challenging group of patients.

\section{Acknowledgments}

The authors thank Jiang Li for his help in statistical analysis.

\section{Disclosure}

Conflicts of interest: None.

\section{References}

1. Tsai TT, Trimarchi S, Nienaber CA. Acute aortic dissection: perspectives from the International Registry of Acute Aortic Dissection (IRAD). Eur J Vasc Endovasc Surg 2009; 37: 149-59.

2. Krüger T, Weigang E, Hoffmann I, Blettner M, Aebert H, GERAADA Investigators. Cerebral protection during surgery for acute aortic dissection type A: results of the German Registry for Acute Aortic Dissection Type A (GERAADA). Circulation 2011; 124: 434-43.

3. Sun L, Qi R, Zhu J, Liu Y, Zheng J. Total arch replacement combined with stented elephant trunk implantation: a new "standard" therapy for type A dissection involving repair of the aortic arch? Circulation 2011; 123: 971-8.

4. Mussa FF, Horton JD, Moridzadeh R, Nicholson J, Trimarchi S, Eagle KA. Acute aortic dissection and intramural hematoma: a systematic review. JAMA 2016; 316: 754-63.

5. Elsayed RS, Cohen RG, Fleischman F, Bowdish ME. Acute type A aortic dissection. Cardiol Clin 2017; 35: 331-45

6. Neri E, Toscano T, Papalia U, et al. Proximal aortic dissection with coronary malperfusion: presentation, management, and outcome. J Thorac Cardiovasc Surg 2001; 121: 552-60.

7. Patel HJ, Williams DM, Dasika NL, Suzuki Y, Deeb GM. Operative delay for peripheral malperfusion syndrome in acute type A aortic dissection: a long-term analysis. J Thorac Cardiovasc Surg 2008; 135: 1288-95.

8. Deeb GM, Williams DM, Bolling SF, et al. Surgical delay for acute type A dissection with malperfusion. Ann Thorac Surg 
1997; 64: 1669-75.

9. Girardi LN, Krieger KH, Lee LY, Mack CA, Tortolani AJ, Isom OW. Management strategies for type A dissection complicated by peripheral vascular malperfusion. Ann Thorac Surg 2004; 77: 1309-14.

10. Sun X, Lu S, Yang S, et al. Open triple-branched stent graft placement for the surgical treatment of acute aortic arch dissection. J Cardiothorac Surg 2012; 7: 130.

11. Lu S, Yang S, Lai H, et al. Open aortic arch reconstruction for acute type A aortic dissection: a single-center experience with 267 consecutive patients. J Cardiothorac Surg 2016; 11: 111.

12. Chen YF, Chien TM, Yu CP, et al. Acute aortic dissection type A with acute coronary involvement: a novel classification. Int J Cardiol 2013; 168: 4063-9.

13. Eren E, Toker ME, Tunçer A, et al. Surgical management of coronary malperfusion due to type A aortic dissection. J Card Surg 2007; 22: 2-6.

14. Hsing CH, Hsieh MY, Chen WY, Cheung So EC, Cheng BC, Chang MS. Induction of interleukin-19 and interleukin-22 after cardiac surgery with cardiopulmonary bypass. Ann Thorac Surg 2006; 81: 2196-201

15. Yellon DM, Hausenloy DJ. Myocardial reperfusion injury. $\mathrm{N}$ Engl J Med 2007; 357: 1121-35.

16. Modi A, Diprose P, Tsang G. Complete avulsion of right coronary artery caused by acute type-A aortic dissection. Heart 2013; 99: 1628.

17. Kawahito K, Adachi H, Murata SI, Yamaguchi A, Ino T. Coronary malperfusion due to type A aortic dissection: mechanism and surgical management. Ann Thorac Surg 2003; 76: 1471-6. 